\title{
Impact de la solidarisation des couronnes implanto-portées sur le remodelage osseux péri-implantaire : étude clinique prospective avec suivi à 5 ans
}

\author{
Thomas Fortin ${ }^{1,5}$, Marion Paris ${ }^{1}$, Sophie Thoret ${ }^{2}$, Nathalie Rancon ${ }^{3}$, \\ Christophe Deschaume ${ }^{4}$, Sandra David-Tchouda ${ }^{5}$
}

1 Département de chirurgie orale, faculté d'odontologie de Lyon. Unité fonctionnelle d'implantologie orale, service de consultation et de traitements dentaires, pôle Nord, Hospices Civils de Lyon, France

${ }^{2}$ Centre d'investigation clinique de Grenoble, INSERM, France

${ }^{3}$ Centre d'investigation clinique de la Croix Rousse, pôle Nord, Hospices Civils de Lyon, France

${ }^{4}$ Département de chirurgie orale, faculté d'odontologie de Clermont-Ferrand, France

${ }^{5}$ Unité d'évaluation médico-économique, CHU Grenoble, France / ThEMAS TIMC UMR CNRS 5525, Université Joseph Fourier, Grenoble, France

(Reçu le 27 décembre 2014, accepté le 20 décembre 2015)

Mots clés :

cratérisation / implant / couronnes solidarisées ou non / implants courts
Key words: bone loss / dental implant / uncoupled or splinted dental implants / short implant

\begin{abstract}
Résumé - Introduction : L'étude présentée cherchait à mettre en évidence un éventuel lien entre la solidarisation des couronnes et la cratérisation osseuse autour de ces implants. Méthodes : il s'agissait d'une étude prospective sur les implants courts pour le traitement des édentements postérieurs libres mandibulaires. Le traitement correspondait à la mise en place de prothèses scellées sur des implants. Le nombre d'implants posés était identique au nombre de dents à remplacer. La perte osseuse autour des implants était mesurée radiologiquement lors de la mise en place chirurgicale puis tous les ans jusqu'à la cinquième année. La comparaison de la perte osseuse moyenne autour des implants solidarisés versus implants non solidarisés était faite au moyen d'un test $t$ de comparaison de deux moyennes avec des variances égales en prenant l'hypothèse qu'il n'y a pas de différence entre les couronnes solidarisées ou non. Résultats : À 5 ans, les données de 89 implants ont été évaluées. $100 \%$ des implants étaient encore en place. La cratérisation était de $0,69 \mathrm{~mm}$ en moyenne (sd. 1.29) et ne semblait pas différente en fonction des paramètres étudiés. Conclusion : Malgré les limites de cette étude, il semblerait qu'il y ait peu d'impact de la solidarisation des couronnes sur la cratérisation péri-implantaire.
\end{abstract}

\begin{abstract}
Effects of prosthesis splinting or not on peri-implant bone loss: a prospective clinical study with a 5-year follow-up period. Introduction: The study presented sought to demonstrate a possible relation between the splinting of crowns on short implants $(6-8 \mathrm{~mm}$ long) placed in the posterior mandibular sector and bone loss around these implants. Materials and methods: The sample of patients participating in this multicenter prospective study included the first 54 patients included in a study on short implants. Their inclusion required that they present a mandibular free posterior gap, with bone height between 8 and $10 \mathrm{~mm}$, as well as a bone ridge greater than $6 \mathrm{~mm}$ above the alveolar canal. Treatment comprised implanting prostheses onto implants, with the number of implants identical to the number of teeth to be replaced. Bone loss around the implants was measured radiologically during surgical placement and then once a year for 5 years. Results: This study included 124 implants placed in 52 patients who received at least one implant and a maximum of six implants. The sex ratio was relatively well balanced, with $56 \%$ females versus $44 \%$ males. At 5 years, based on the number of patients presenting at the follow-up visit, 89 implants were assessed. One hundred percent of the implants placed were still in place. Peri-implant bone loss was a mean $0.69 \mathrm{~mm}$ (SD 1.29). Mean bone loss around the splinted implants
\end{abstract}

\footnotetext{
* Correspondance : thomas.fortin@univ-lyon1.fr
}

This is an Open Access article distributed under the terms of the Creative Commons Attribution License (http://creativecommons.org/licenses/by/4.0), which permits unrestricted use, distribution, and reproduction in any medium, provided the original work is properly cited 
versus non-splinted implants was compared using a $t$-test comparing the two means with equal variances based on the hypothesis that there would be no difference between splinting and non-splinting crowns. It seems there were few significant differences in terms of bone loss whether or not the crowns were splinted. Conclusion: Despite the limitations of this study, the small sample and post-hoc analysis, we obtained a preliminary quantitative assessment that splinting prostheses seems to have little impact on peri-implant bone loss.

\section{Introduction}

Les implants dentaires sont aujourd'hui complètement intégrés dans l'arsenal thérapeutique du chirurgien-dentiste. Depuis les implants de Bränemark, à la fin des années 1970, l'évolution a été constante. La mise en place d'implants dans des secteurs anatomiques parfois défavorables a conduit au développement des implants courts. Ces solutions bien documentées, moins invasives que les techniques de greffes osseuses, sont de plus en plus utilisées [1-5]. Le problème de cratérisation osseuse autour des implants courts est un paramètre important. Perdre $2 \mathrm{~mm}$ d'os sur un implant de $6 \mathrm{~mm}$ peut sembler plus préjudiciable que pour un implant de $10 \mathrm{~mm}$. Divers facteurs étiologiques sont évoqués pour expliquer la cratérisation : la chaleur générée au cours du forage, la pression exercée pour la mise en place de l'implant, la quantité osseuse autour de l'implant inférieur à 1,5 mm [6], la qualité de l'ostéointégration [7], le diamètre, le nombre d'implants et leur orientation [8], les surcharges occlusales [9], un défaut de maintenance [10] ou bien encore le type de connexion prothétique [11]. Pour diminuer les surcharges occlusales, et ainsi les contraintes mécaniques transmises aux implants, il a été proposé de solidariser les implants entre eux [12]. En effet, le fait de solidariser les implants augmenterait la surface sur laquelle la contrainte se distribue, diminuant ainsi la transmission de la contrainte mécanique aux structures osseuses [13]. Cette hypothèse est contredite par Blanes et ses collaborateurs (2007) qui ont suivi 192 implants sur une période allant de 4 à 10 ans et qui ne notent aucune différence de remodelage osseux autour des implants entre les implants solidarisés et ceux qui ne le sont pas. Dans ce suivi longitudinal, tous les implants ont été pris en compte, quel que soit leur longueur, leur positions anatomiques et l'existence ou non de dents distales à la réhabilitation prothétique.

L'objectif de notre étude était d'étudier la relation qui pourrait exister entre le remodelage osseux péri-implantaire et le fait de solidariser ou non les implants de 6 à $8 \mathrm{~mm}$ de longueur placés dans la région mandibulaire postérieure sans dent naturelle distale à la réhabilitation prothétique.

\section{Matériel et méthode}

Une étude prospective de cohorte a été conduite de juillet 2006 à janvier 2013 dans le département d'implantologie orale des Hospices Civils de Lyon (France). Pour les besoins de l'étude présentée, tous les patients ont été considérés sauf ceux ayant été traités par un implant unitaire. (a)

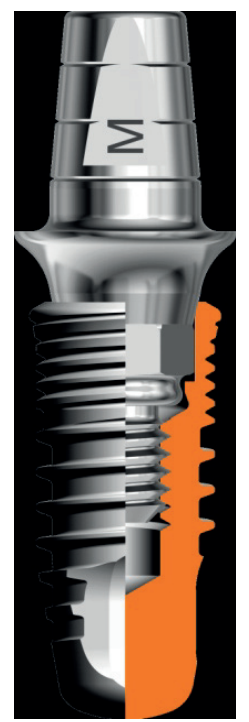

(b)

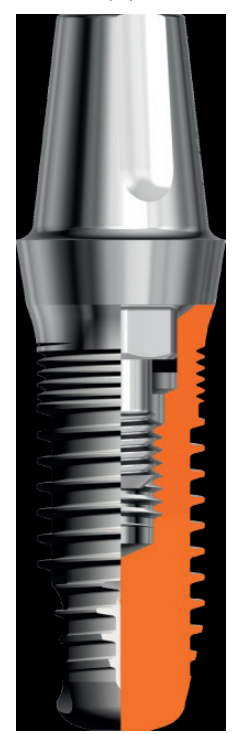

Fig. 1. Illustration schématique des implants utilisés. (a) CLINIC ${ }^{\circledR}$ implant avec une connexion interne et switching platform. (b) EVL PLUS $^{\circledR}$ implant avec un col usiné de $1,2 \mathrm{~mm}$.

Fig. 1. Schematic drawings illustrating the implant-abutment connections. (a) CLINIC ${ }^{\circledR}$ implant with an internal connection, switching platform. (b) EVL PLUS ${ }^{\circledR}$ implant with a 1.2 machined collar, internal connection.

Tous les praticiens participant à l'étude étaient considérés comme experts en implantologie et connaissaient parfaitement le système utilisé.

Les patients ont reçu pour moitié des implants CLINIC ${ }^{\circledR}$ (Global D, Serf, Brignais, France) à switching platform et l'autre moitié des EVL PLUS ${ }^{\circledR}$ (Global D, Serf, Brignais, France) à connexion supracrestale sans switching platform (Fig. 1). Ces deux types d'implants avaient un état de surface identique, sablage par des particules d'alumine pures. Les reconstitutions prothétiques ont toutes été scellées.

Tous les patients ont été informés de l'étude et ont accepté de donner par écrit leur consentement éclairé. L'étude a été approuvée par le comité d'éthique de notre établissement (Hospices Civils de Lyon, $\mathrm{N}^{0}$ 08.183, 15 mai 2008).

\section{Critères d'inclusion}

- Patients ayant donné par écrit leur consentement éclairé. - Patients âgés de plus de 18 ans. 
- Patients présentant une indication de mise en place de un ou plusieurs implants dentaires dans le secteur mandibulaire postérieur avec une hauteur de crête comprise entre 8 et $10 \mathrm{~mm}$ ainsi qu'une largeur de crête osseuse supérieure à $6 \mathrm{~mm}$ au-dessus du canal alvéolaire inférieur.

- Aucune avulsion n'a été pratiquée dans le secteur considéré les trois mois précédant l'étude.

- Patients présentant un schéma occlusal permettant la réalisation de la prothèse sans contact dans les mouvements de latéralité.

- Patients ne présentant pas de parafonction type bruxisme.

\section{Critères de non-inclusion}

- Patients inaptes à comprendre les informations données par le praticien pour des raisons légales, psychiques ou linguistiques.

- Difficultés de suivi (mutation imminente, motivation insuffisante).

- Femmes enceintes ou patient mineur.

- Patients portant une prothèse adjointe mandibulaire pendant les quatre mois de la phase d'ostéointégration.

- Patients présentant un volume osseux insuffisant, apprécié sur le scanner, ne permettant pas la mise en place d'implant.

- Patients ne présentant pas un édentement mandibulaire postérieur libre.

- Patients ne présentant pas un schéma occlusal permettant la réalisation de la prothèse sans contact dans les mouvements de latéralité.

- Patients présentant des parafonctions de type bruxisme.

- Patients présentant un risque d'endocardite infectieuse, de transmission de la maladie de Creutzfeld-Jakob.

- Patients présentant un déficit immunitaire grave ou acquis.

- Patients présentant une hémopathie grave, une hémophilie, une insuffisance rénale chronique, une maladie autoimmune, une affection nécessitant ou devant nécessiter une transplantation d'organes, un diabète mal contrôlé, une ostéoporose, une polyarthrite rhumatismale ou une maladie psychiatrique.

- Patients sous antimitotiques ou immunosuppresseurs, sous corticoïdes à fortes doses.

- Patients toxicomanes, éthylo-tabagiques.

\section{Procédure de mise en place chirurgicale des implants et des prothèses}

Une évaluation de la hauteur d'os disponible était faite en préopératoire, sans utilisation systématique du scanner. Le choix était laissé à l'appréciation du praticien selon ses habitudes cliniques. La chirurgie se faisait sous anesthésie locale (primacaïne adrénalinée 1/100 000). Un lambeau était systématiquement réalisé pour avoir une bonne appréciation de la surface de la crête osseuse. En fonction de la présence ou non de gencive attachée, l'incision était faite sur la crête ou légèrement décalée en lingual de manière à garantir un bandeau de gencive autour de la vis de cicatrisation. Si cela n'était pas suffisant, nous avions recours à une chirurgie mucogingivale avant la mise en place des implants.

Pour les implants « switching platform », le col de l'implant se situait à $0,5 \mathrm{~mm}$ en dessous du sommet de la crête selon les recommandations de la société Global D. Pour les implants à connexion supra-osseuse, le col de l'implant se situait 1,2 mm au-dessus du sommet de la crête selon les recommandations de la société Global $D$. Nous utilisions une technique en un temps chirurgical. Durant toute la période de cicatrisation, le port de prothèse amovible transitoire était interdit. La prémédication était systématique. Elle incluait :

- $2 \mathrm{~g} \mathrm{~d}$ d'amoxicilline une heure avant l'intervention puis en deux prises pendant 6 jours. En cas d'allergie à la pénicilline, la clindamycine était envisageable ;

- corticoïdes : $60 \mathrm{mg}$ le matin de l'intervention puis pendant 3 jours ;

- paracétamol : $1000 \mathrm{mg}$ en début d'intervention puis à la demande sans dépasser 4 par jour ;

- chlorexidine $(0,12 \%)$ en bain de bouche, 3 fois par jour pendant 10 jours.

La reprise de l'hygiène se faisait le jour même avec l'utilisation d'une brosse à dent chirurgicale (7/100).

Les couronnes furent scellées sur les implants, sans connexion avec les dents naturelles. Elles furent solidarisées ou non, à la convenance du praticien. Le réglage de l'occlusion fut réalisé de manière à obtenir un contact léger en intercuspidie maximale et une absence de contact en latéralité, travaillante et non travaillante. Le nombre d'implants posés était identique au nombre de dents à remplacer. Dans l'étude, nous n'avons pris en compte que les implants de 6 ou $8 \mathrm{~mm}$ en position distale.

Les visites de suivi ont été effectuées par le praticien qui a opéré le patient à différentes étapes : (0) immédiatement après la chirurgie ; (1) une semaine après la chirurgie pour l'ablation des sutures ; $(2)$ quatre mois après la chirurgie pour la mise en charge ; (3) puis tous les ans pendant cinq ans. À chaque visite, une radiographie intra-orale a été réalisée.

\section{Analyse du remodelage osseux péri-implantaire}

Pour étudier le remodelage osseux péri-implantaire, seule l'analyse radiologique a été prise en compte. Le niveau osseux (NO), contact os-implant le plus coronal, autour de l'implant était déterminé à l'aide de téléradiographies intrabuccales (TIB), nommées aussi rétroalvéolaires. La méthode employée était celle des plans parallèles. Le film radiographique (numérique ou Kodak Ektaspeed Plus film pour limiter l'exposition des patients aux rayonnements ionisants) était placé dans un angulateur de RINN. Pour la prise des clichés, l'angulateur était mis en bouche, film parallèle à l'axe des implants. La source 

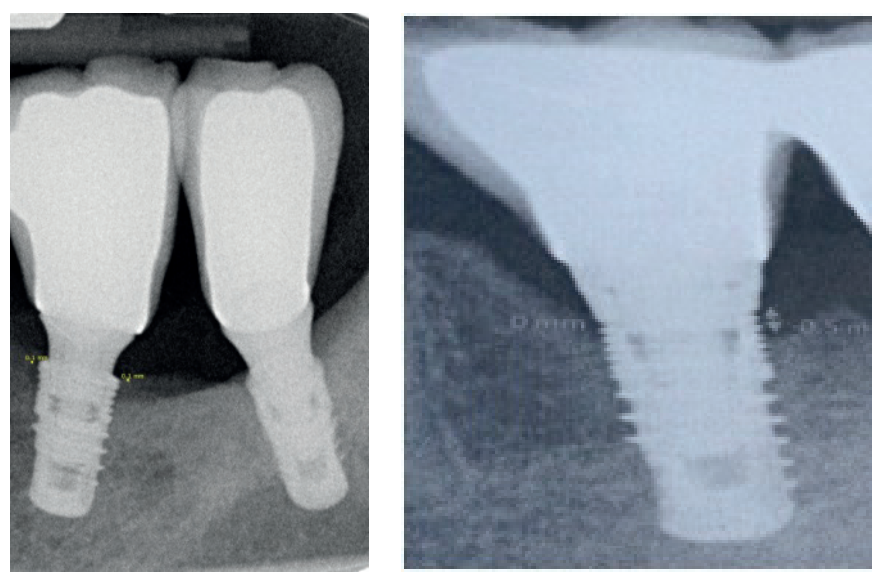

Fig. 2. Position du point de référence sur l'implant CLINIC ${ }^{\circledR}$ (gauche) et EVL PLUS ${ }^{\circledR}$ (droite).

Fig. 2. Reference point on the implant Clinic ${ }^{\circledR}$ (left) and EVL PLUS ${ }^{\circledR}$ (right).

des rayons $X$ était perpendiculaire au film. Un cliché était considéré comme bon pour l'étude si les spires de l'implant n'apparaissaient pas superposées. Un cliché radiographique ne devait contenir que deux dents à analyser. La mesure du NO était réalisée en mésial et en distal de l'implant, à la verticale de celui-ci. Pour les implants CLINIC, le point de référence était le sommet de l'implant. Une valeur positive correspondait à un NO situé au-dessus du point de référence. Au contraire, un niveau osseux négatif était situé en dessous du point de référence. Pour les implants EVL PLUS, le point de référence était la jonction entre le col lisse, non ostéointégré et la partie endoosseuse (Fig. 2).

\section{Analyse statistique}

Les statistiques descriptives incluaient pourcentage et fréquences pour les variables non continues avec un intervalle de confiance à $95 \%$, et moyenne et déviations standard pour les variables continues. Un test de $\mathrm{Chi}^{2}$ (le test de Fisher si nécessaire) a été utilisé pour les variables non continues. Pour la comparaison de la perte osseuse moyenne des implants solidarisés versus implants non solidarisés, nous avons réalisé un test $t$ de comparaison de deux moyennes qui ont des variances égales. Toutes les analyses statistiques ont été effectuées en utilisant le logiciel Stata SE version 11.0 software (StataCorp LP, College Station, TX, USA, www.stata.com).

\section{Résultats}

\section{Description de la population étudiée}

Un total de 54 patients a été inclus dans cette étude pour une période de suivi de cinq ans. Deux patients ne se sont pas présentés pour la mise en place chirurgicale des implants. Ainsi 52 patients (29 femmes et 23 hommes) ont finalement participé à l'étude avec un âge moyen de $57 \pm 8$ ans pour la mise en place de 122 implants (Tab. I). Un patient avec un implant unique ne s'est pas présenté pour l'étape prothétique. Chaque patient a été rappelé pour être incité à venir aux différentes visites de contrôle. Cinq ans après la pose des prothèses, 41 patients se sont présentés à la visite de contrôle (Fig. 3). Concernant l'analyse par implant, certaines radiographies n'étaient pas totalement exploitables. Ces données manquantes ont exclu les implants de l'analyse statistique à l'étape considérée. En termes d'âge et de sexe, il n'y avait pas de différence significative entre le groupe de patients « perdus de vue » et le groupe de patients considérés dans l'analyse. Les deux types d'implants posés étaient représentés de manière égale : $51 \%$ des implants de type EVL PLUS $(n=63)$ et $48 \%$ de type CLINIC $(n=60)$. À 5 ans, nous avions des informations sur 41 implants EVL PLUS et 54 implants CLINIC.

\section{Remodelage osseux péri-implantaire (Tab. II)}

La comparaison de la perte osseuse moyenne entre les implants solidarisés et non solidarisés, côté distal puis côté mésial, est présentée Tableau III et Tableau IV. Pour le remodelage osseux distal, nous obtenons $p=0,5567$. Nous n'avons donc pas de différence de perte osseuse, que les couronnes soient solidarisées ou pas pour les mesures prises en distal de l'implant. Pour le remodelage osseux mésial, nous obtenons $p=0,0184$. La valeur de $p$ est ici très inférieure à $5 \%$, ce qui signifie qu'il y aurait une différence significative de perte osseuse mésiale entre les couronnes solidarisées et les couronnes non solidarisées. On remarque en effet dans le tableau que la cratérisation moyenne en $\mathrm{mm}$ est supérieure pour les couronnes non solidarisées mais reste minime.

Le remodelage osseux péri-implantaire en comparant le côté mésial avec le côté distal pour des couronnes non solidarisées puis pour des couronnes solidarisées est donné Tableaux V et VI. En partant de l'hypothèse qu'il n'y a pas de différence entre les deux côtés, nous obtenons respectivement pour les couronnes non solidarisées et solidarisées, $p=0,3699$ et $p=0,3225$. Ces deux résultats étant supérieurs à $5 \%$, il n'y a pas de différence significative entre les deux côtés, que les implants soient solidarisés ou non.

\section{Discussion}

Le succès implantaire est associé à un remodelage osseux. La perte osseuse acceptable est un concept qui a évolué avec le temps [15]. Quels que soient les facteurs qui influencent cette perte osseuse, et bien qu'il n'existe pas de consensus sur la quantité osseuse et sa progression dans le temps après la mise en charge [16], des études cliniques mettent en évidence 
Tableau I. Description de la population à l'inclusion en fonction du type d'implant.

Table I. Characteristics of patients at inclusion according to the implant type.

\begin{tabular}{lccc}
\hline & CLINIC & EVL & $p$-value \\
\hline Sex (males, \%) & $48.2 \%$ & $38.5 \%$ & NS* $^{*}$ \\
Age (mean \pm SD) & $57 \pm 8.8$ & $58 \pm 7.1$ & NS \\
\hline
\end{tabular}

* Fisher exact test

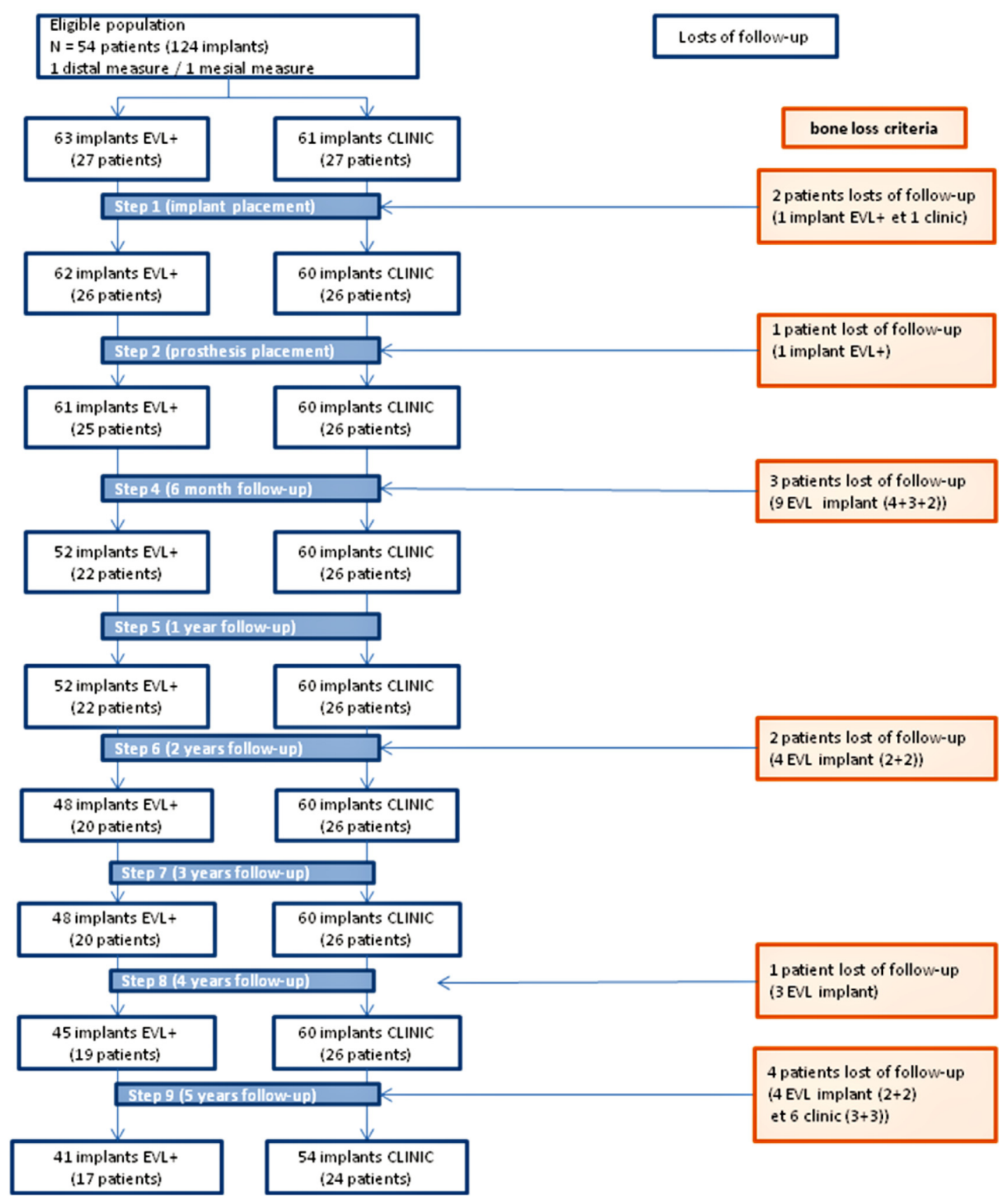

Fig. 3. Processus détaillant les recrutements, calendrier des événements, allocations et sorties d'études. Fig. 3. flowchart detailing subject recruitment, timetable, measurements, allocation and dropout. 
Tableau II. Niveau osseux (N0) par rapport au point de référence défini sur l'implant.

Table II. Bone level around the implant.

\begin{tabular}{|l|c|c|c|c|c|}
\hline Variable & Nombre d'implants & $\begin{array}{c}\text { Niveau osseux moyen } \\
(\mathrm{mm})\end{array}$ & Std. Dev. & $\begin{array}{c}\text { Minimum } \\
(\mathrm{mm})\end{array}$ & Maximum (mm) \\
\hline Chirurgie & 122 & .3214912 & .6155884 & -1.3 & 1.8 \\
\hline Prothèse & 121 & -.463964 & .7608659 & -3.1 & 1.1 \\
\hline 5 ans & 95 & -.6988764 & 1.29202 & -5 & 1.7 \\
\hline
\end{tabular}

Tableau III. Perte osseuse péri-implantaire en cas de solidarisation (S) versus non-solidarisation (NS) côté distal. Table III. Bone loss surrounding uncoupled (S) or splinted (NS) dental implants: distal position.

\begin{tabular}{|l|l|l|l|l|l|}
\hline Group & Obs & Moyenne $(\mathrm{mm})$ & Écart type $(\mathrm{mm})$ & \multicolumn{3}{|l|}{ intervalle de confiance à $95 \%$} \\
\hline N.S. & 32 & -.146875 & 1.426022 & -.6610107 & .3672607 \\
S & 50 & .018 & 1.095387 & -.2933056 & .3293056 \\
\hline Combined & 82 & .0463415 & 1.229091 & -.3164024 & .2237195 \\
\hline
\end{tabular}

Tableau IV. Perte osseuse péri-implantaire en cas de solidarisation (S) versus non-solidarisation (NS) côté mésial. Table IV. Bone loss surrounding uncoupled (S) or splinted (NS) dental implants: mesial position.

\begin{tabular}{|l|l|l|l|l|l|}
\hline Group & Obs & Moyenne $(\mathrm{mm})$ & Écart type $(\mathrm{mm})$ & \multicolumn{3}{|l|}{ Intervalle de confiance à 95 \% } \\
\hline N.S & 33 & -.4848485 & 1.584322 & -1.046625 & .0769276 \\
\hline S & 49 & .2428571 & 1.154159 & -.0886561 & .5743704 \\
\hline Combined & 82 & -.05 & 1.382005 & -.3536598 & .2536598 \\
\hline
\end{tabular}

Tableau V. Perte osseuse péri-implantaire du côté mésial versus côté distal avec des couronnes non solidarisées. Table V. Bone loss surrounding implant, mesial versus distal position with splinted (NS) dental implants.

\begin{tabular}{|l|l|l|l|l|l|}
\hline Variable & Obs & Moyenne $(\mathrm{mm})$ & Écart type $(\mathrm{mm})$ & \multicolumn{3}{|l|}{ Intervalle de confiance à 95 \% } \\
\hline Mésial & 33 & -.4848485 & 1.584322 & -1.046625 & .0769276 \\
\hline Distal & 32 & -.146875 & 1.426022 & -.6610107 & .3672607 \\
\hline Combined & 65 & - & 1.50633 & - & .0547887 \\
\hline
\end{tabular}

Tableau VI. Perte osseuse péri-implantaire du côté mésial versus côté distal avec des couronnes solidarisées.

Table VI. Bone loss surrounding implant, mesial versus distal position with uncoupled dental implants.

\begin{tabular}{|l|l|l|l|l|l|}
\hline Variable & Obs & Moyenne $(\mathrm{mm})$ & Écart type $(\mathrm{mm})$ & \multicolumn{3}{|l|}{ intervalle de confiance à $95 \%$} \\
\hline Mésial & 49 & .2428571 & 1.154159 & -.0886561 & .5743704 \\
\hline Distal & 50 & .018 & 1.095387 & -.2933056 & .3293056 \\
\hline Combined & 99 & .1292929 & 1.12479 & -.0950426 & .3536284 \\
\hline
\end{tabular}

un remodelage de $1,10 \mathrm{~mm}$ la première année puis une moyenne de 0,1 mm par an sur un suivi de cinq ans $[17,18]$. Ces observations ont été faites sur des implants d'une longueur de $10 \mathrm{~mm}$ et plus. Les résultats radiologiquement constatés à cinq ans dans cette étude sur des implants courts (cf. Tab. I) confirment ces études.

La solidarisation des implants a été recommandée pour permettre une meilleure répartition des forces et une diminution du stress subi par l'os, donc la cratérisation [3, 19, 20]. Lorsque des forces angulées sont appliquées, l'étude de Bergkvist et al. (2008) [21] montre que le fait de solidariser rigidement les implants contigus diminue le stress transmis à l'os. Wang et al. (2002) [22] parviennent aux mêmes conclusions, pour un os de mauvaise qualité. La lecture des résultats de l'étude présentée ici est plus mitigée. Il convient d'interpréter avec prudence la différence significative que l'on trouve au niveau mésial. En effet, on ne peut en expliquer le mécanisme physiopathologique, la cohorte reste d'effectif modeste et il s'agit d'une analyse post hoc puisque les données sont extraites d'une étude dont le dessin a été fait en fonction d'un objectif principal différent. Ce fait nécessite, pour être validé, d'être étayé par une étude étiologique spécifiquement conçue 
dans cet objectif. Malgré les limites de cette étude, nous obtenons une première évaluation quantitative de l'impact de la solidarisation des implants de 6 à $8 \mathrm{~mm}$ de long en secteur mandibulaire postérieur sans dent naturelle distale sur le remodelage osseux : il semblerait qu'il y ait peu d'impact sur la cratérisation péri-implantaire. Ceci va dans le sens des résultats de Blanes et al. (2007) [14]. Leur étude diffère de celle-ci en ce sens qu'elle considère l'influence de la solidarisation dans les secteurs postérieurs mandibulaire et maxillaire et que seule la moitié des implants correspondent à la définition que nous avons choisie pour les implants courts. De plus, les restaurations prothétiques peuvent associer à la fois des implants courts et des implants longs.

L'impact de la solidarisation sur le remodelage osseux pourrait ne pas être dû à la solidarisation en tant que telle mais à l'absence de passivité des armatures source de transmission de contraintes mécaniques à l'os [23, 24]. De plus, les facteurs biologiques sont prépondérants. La présence d'un hiatus entraîne l'apparition de bactéries provoquant un risque d'infection, mais aussi la formation d'une épine irritative déclenchant une inflammation $[19,25]$. Les couronnes unitaires facilitent l'accès à l'hygiène pour le patient [23, 26] et elles sont plus faciles à démonter ou à changer en cas de besoin surtout pour les couronnes scellées. Elles limitent l'apparition de complications prothétiques liées aux éventuelles forces de flexion de la mâchoire [27]. Pour autant, le réglage du point de contact constitue une difficulté. Il est préférable de solidariser les couronnes contiguës plutôt que d'obtenir des points de contact trop forts [28], qui empêchent la parfaite mise en place des couronnes entraînant la formation d'un hiatus, favorisant l'inflammation et l'infection à l'origine d'une cratérisation.

Conflits d'intérêt : La société Global D a fourni gracieusement les implants. Aucun des investigateurs n'a jamais reçu de rémunération de la part de la société Global $D$, il n'existe aucun conflit d'intérêt.

Remerciements : Les auteurs souhaitent remercier les docteurs Alain Laguilhomie, Michel Isidori, Hervé Bouchet, Charles Coudurier, Christophe Deschaume pour leur aide.

\section{Références}

1. Lum LB. A biomechanical rationale for use of short implants. J Oral Implantol 1991;17:126-131.

2. Mish CE. Short dental implants: a literature review and rationale for use. Dent Today 2005;24:64-68.

3. Misch CE, Steigenga J, Barboza E, Misch-Dietsh F, Canciola LJ, Kazor C. Short dental implants in posterior partial edentulism: a multicenter retrospective 6 -year case series study. J Periodontol 2006;77:1341-1347.

4. Tawil G, Aboujaoude N, Younan R. Implants courts: les taux de survie et de complications. Titane 2006;3:43-50.
5. Grant BT, Pancko FX, Kraut RA. Outcomes of placing short dental implants in the posterior mandible: A retrospective study of 124 cases. J Oral Maxillofac Surg 2009;67:713-717.

6. Davarpanah M, Szmuklr-Moncler S, Khoury PM, Jabubowicz-Kohen B, Martinez H. Manuel d'implantologie clinique: Concepts, protocoles et innovations recentes. $2^{\mathrm{e}}$ ed. CdP, Paris, 2008.

7. Hoff L. Influence des traitements de surface implantaire sur l'accélération de l'ostéo intégration. Thèse Chir Dent. Nancy : Univ H. Poincaré, 2012.

8. Nissan J, Gross 0, Ghelfan 0, Priel I, Gross M, Chaushu G. The effect of splinting implant-supported restorations on stress distribution of different crown-implant ratios and crown height spaces. J Oral Maxillofac Surg 2011;12:2990-2994.

9. Isidor F. Histological evaluation of peri-implant bone on implant subjected to overload or plaque accumulation. Clin Oral Implants Res 1997;8:1-9.

10. Isidor F. Loss of osseointegration caused by occlusal load of oral implants. A clinical and radiographic study in monkeys. Clin Oral Implants Res 1996;7:143-152.

11. Canullo L, Fedele GR, Iannello G, Jepsen S. Platform switching and marginal bone-level alterations: the results of a randomizedcontrolled trial. Clin Oral Implants Res 2010;21:115-121.

12. Serio FG. Clinical rationale for tooth stabilization and splinting. Dent Clin North Am 1999;43:1-12.

13. Brunski JB, Puleo DA, Nanci A. Biomaterials and biomechanics of oral and maxillofacial implants: Current status and future developments. Int J Oral Maxillofac Implants 2000;15:15-21.

14. Blanes RJ, Bernard JP, Blanes ZM, Belser UC. A 10-year prospective study of ITI dental implants placed in the posterior region. II: Influence of the crown-to-implant ratio and different prosthetic treatment modalities on crestal bone loss. Clin Oral Impl Res 2007;18:707-714.

15. Valderrama P, Jones AA, Wilson TG, Higginbottom F, Schoolfield $J D$, Jung RE, et al. Bone changes around early loaded chemically modified sandblasted and acid-etched surfaced implants with and without a machined collar: a radiographic and resonance frequency analysis in the canine mandible. Int J Oral Maxillofac Implants 2010;25:548-557.

16. Monje A, Suarez F, Galindo-Moreno P, Garcia-Nogales A, Fu J-H, Wang HL. A systematic review on marginal bone loss around short dental implants $(<10 \mathrm{~mm})$ for implant supported fixed prostheses. Clin Oral Implants Res 2014;25:1119-1124.

17. Cochran DL, Bosshardt DD, Grize L, Higginbottom FL, Jones AA, Jung $\mathrm{RE}$, et al. Bone response to loaded implants with non-matching implant-abutment diameters in the canine mandible. J Periodontology 2009;80:609-617.

18. Hartman $\mathrm{GA}$, Cochran DL. Initial implant position determines the magnitude of crestal bone remodeling. J Periodontology 2004; 75:572-577.

19. Skalak R. Biomechanical considerations in osseointegrated prostheses. J Prosthet Dent 1983;49:843-848.

20. Rangert $B$, Jemt $T$, Jörneus L. Forces and moments on Branemark implants. Int J Oral Maxillofacial Implants 1989;4:241-247.

21. Bergkvist G, Simonsson K, Rydberg K, Johansson F, Dérand T. A finite element analysis of stress distribution in bone tissue 
surrounding uncoupled or splinted dental implants. Clin Implant Dent Relat Res 2008;10:40-46.

22. Wang IM, Teu LJ, Wang J, Lin LD. Effects of prosthesis materials and prosthesis splinting on peri-implant bone stress around implants in poor quality bone: a numeric analysis. Int J Oral Maxillofac Implants 2002;17:231-237.

23. Smith DE, Zarb GA. Criteria for success of Osseointegrated endosseous implants. J Prosthet Dent 1989;62:567-572.

24. Rasmusson L, Kahnberg KE, Tan A. Effects of implant design and surface on bone regeneration and implant stability: an experimental study in the dog mandible. Clin Implant Dent Relat Res 2001;3:2-8.
25. Wyatt CC, Zarb GA. Treatment outcomes of patients with implantsupported fixed partial prostheses. Int J Oral Maxillofac Implants 1998;13:204-211.

26. Grossmann Y. Indication for splinting implant restorations. J Oral Maxillofac Surg 2005;63:1642-1652.

27. Kregzde M. A method of selecting the best implant prosthesis design option using three-dimensional finite element analysis. Int J Oral Maxillofac Implants 1993;8:662-673.

28. Guichet DL, Caputo AA, Yoshinobu D, David L. Effect of splinting and interproximal contact tightness on load transfert by implant restorations. J Prosthet Dent 2002;87:528-535. 